OPEN ACCESS

Edited by: Andriy Myachykov, Northumbria University, United Kingdom

Reviewed by:

Kathrin Rothermich,

East Carolina University, United States Lisa Goffman

The University of Texas at Dallas, United States

*Correspondence:

Eleonora J. Beier ejbeier@ucdavis.edu

Specialty section: This article was submitted to Cognition,

a section of the journal

Frontiers in Psychology

Received: 26 November 2017

Accepted: 15 March 2018

Published: 03 April 2018

Citation:

Beier EJ and Ferreira F (2018) The

Temporal Prediction of Stress in Speech and Its Relation to Musical Beat Perception.

Front. Psychol. 9:431. doi: 10.3389/fpsyg.2018.00431

\section{The Temporal Prediction of Stress in Speech and Its Relation to Musical Beat Perception}

\author{
Eleonora J. Beier* and Fernanda Ferreira \\ Department of Psychology, University of California, Davis, Davis, CA, United States
}

While rhythmic expectancies are thought to be at the base of beat perception in music, the extent to which stress patterns in speech are similarly represented and predicted during on-line language comprehension is debated. The temporal prediction of stress may be advantageous to speech processing, as stress patterns aid segmentation and mark new information in utterances. However, while linguistic stress patterns may be organized into hierarchical metrical structures similarly to musical meter, they do not typically present the same degree of periodicity. We review the theoretical background for the idea that stress patterns are predicted and address the following questions: First, what is the evidence that listeners can predict the temporal location of stress based on preceding rhythm? If they can, is it thanks to neural entrainment mechanisms similar to those utilized for musical beat perception? And lastly, what linguistic factors other than rhythm may account for the prediction of stress in natural speech? We conclude that while expectancies based on the periodic presentation of stresses are at play in some of the current literature, other processes are likely to affect the prediction of stress in more naturalistic, less isochronous speech. Specifically, aspects of prosody other than amplitude changes (e.g., intonation) as well as lexical, syntactic and information structural constraints on the realization of stress may all contribute to the probabilistic expectation of stress in speech.

Keywords: rhythm, stress, speech, music, meter, prediction, prosody, language

\section{INTRODUCTION}

In the domain of music, it is well established that metric structure gives rise to expectancies which allow humans to perceive and synchronize to a beat (Large and Kolen, 1994; Large and Jones, 1999). As music and language share many cognitive mechanisms and neural resources (Patel, 2008), the same beat perception mechanisms applied to musical rhythm might also be used when processing speech rhythms, involving the representation of a metrical structure and prediction of where the next stressed syllable will occur. Rhythmic properties of speech based on the alternation of stressed and unstressed syllables have been posited to be organized into hierarchical metrical trees or grids, which may be similar to musical meter (e.g., Martin, 1972; Liberman and Prince, 1977; Selkirk, 1984; Ferreira, 1993, 2007). However, while prediction mechanisms are thought to underlie many aspects of language processing (Federmeier and Kutas, 1999; Pickering and Garrod, 2007; Kuperberg and Jaeger, 2016), it is less clear to what extent rhythmic features of speech are predicted during on-line comprehension. 
While rhythmic perception in music operates on periodic, hierarchically organized beats, the same periodicity is seldom found in naturalistic speech (Lehiste, 1977; Dauer, 1983). Thus, while still presenting rhythmic properties, the stress patterns of speech may typically be too varied to give rise to meaningful expectations (Patel, 2008; London, 2012). On the other hand, there are several reasons why predicting when the next stressed syllable will occur in speech may be useful. Stress patterns are an important segmentation cue in speech and in language acquisition (Sanders and Neville, 2000; Nazzi and Ramus, 2003), and aid word recognition in the presence of many competing lexical items (Norris et al., 1995). Thus, successful prediction of the occurrence of stresses may aid the difficult task of breaking the continuous speech signal into its components.

The temporal prediction of stress could also be beneficial by reducing processing costs during language comprehension. Stressed syllables are detected and processed faster than unstressed ones (Cutler and Foss, 1977; Gow and Gordon, 1993). Crucially, shorter reaction times (RTs) are also found when an acoustically identical phoneme is predicted to be stressed based on various aspects of the preceding context (Cutler, 1976; Cutler and Fodor, 1979; Pitt and Samuel, 1990). Stressed syllables are thought to carry higher informational content than unstressed ones (Altman and Carter, 1989), and stress patterns appear to be strongly related to the information structure of a sentence (Aylett and Turk, 2004; Calhoun, 2010). Therefore, in order to more easily integrate new information, listeners may predict the timing of future stresses and allocate attention and processing resources to those points in time. This has been termed the Attentional Bounce Hypothesis ( $\mathrm{ABH}$ ) (Pitt and Samuel, 1990). The theoretical background surrounding this hypothesis is discussed below. We then turn to evidence for and against temporal prediction of stress in speech, its possible neural mechanisms, and its limitations.

\section{HOW ARE PREDICTIONS FORMED?}

Most work on the prediction of stress in speech assumes that listeners form expectations based on perceived regularities in the stress pattern of a sentence. Originally, through the definition of English as a "stress-timed" language, regularity was thought to consist of the physical periodicity of stresses occurring at close to isochronous intervals (Abercrombie, 1967), which would easily lead a listener to infer the next occurrence of a stress. However, naturalistic speech does not present this degree of periodicity (Lehiste, 1977). While the periodicity of stress may be primarily a perceptual phenomenon (Lehiste, 1977), as captured in the notion of perceptual centers (p-centers; Morton et al., 1976), this claim is controversial, and there is no consensus regarding the presence of isochrony either in the signal or as a perceptual experience.

Regularities in speech stress patterns have also been characterized through hierarchical metrical trees or grids. This concept comes from phonological theories designed to explain how stress is distributed in a sentence (e.g., Martin, 1972; Liberman and Prince, 1977; Hayes, 1983; Selkirk, 1984). A basic tenet of most theories is the avoidance of stress clashes or lapses (two stressed or two unstressed syllables next to each other; e.g., thirTEEN turns into THIRteen MEN), which in practice renders the pattern of stresses more periodic (Selkirk, 1984). However, these theories focus primarily on the hierarchical nature of stress structure and not on periodicity (Martin, 1972). The timing and prominence of every event in a sequence is determined by that of all other sounds through an internal, hierarchical structure, in opposition to sounds concatenated at a single level, as in the case of a simple isochronous beat.

Martin (1972) proposed that listeners internalize this hierarchical structure during on-line comprehension and can thus predict the location of future stresses. This in turn would allow them to allocate their attention to those points in time, facilitating processing, a concept that was later termed the $\mathrm{ABH}$ (Pitt and Samuel, 1990). However, as we will argue, research following this proposal focused less on hierarchical stress structure and more on expectancies based on periodicity. While Martin frames these as very different types of predictions, the two are not always distinguished in the literature.

We believe much of this confusion arises from an inconsistency in the way terms such as "rhythm" and "meter" are defined. For the sake of clarity we adopt the following definitions, though we acknowledge they are not necessarily the only or best ways to interpret these terms. We view rhythm as an informal way to refer to temporal patterning of events, whereas we treat meter as a specific type of structure. Based on Patel (2011), we define meter as a "hierarchical organization of beats in which some beats are perceived as stronger than others" (Patel, 2011, $100)$, where beats may be constituted by the accents or stresses found in both music and language. Importantly, this definition highlights the hierarchical nature of metric structure and it is thus more in line with theories of metrical grids described above. While this type of structure may tend toward periodicity, such as through stress shift, its realization need not necessarily be periodic.

\section{EVIDENCE}

Several early studies of the prediction of stress in speech utilized phoneme monitoring as an indication of processing speed (e.g., Shields et al., 1974; Cutler, 1976; Pitt and Samuel, 1990; Quené and Port, 2005). Shields et al. (1974) found shorter RTs to phonemes belonging to stressed syllables of nonsense words as opposed to when the same syllables were unstressed. The nonsense words were embedded in sentences, based on the idea that a sentence's stress pattern induces timing expectancies for future stresses (Martin, 1972). However, this experiment did not entirely rule out the possibility that the acoustic saliency of stressed syllables, rather than their temporal predictability, may have facilitated processing (Cutler, 1976; Pitt and Samuel, 1990). Such acoustic differences were controlled for in a subsequent test of the ABH (Pitt and Samuel, 1990, Exp. 1). Two-syllable words which could be accented on the first or second syllable (verb-noun pairs such as PERmit vs. perMIT) were embedded within sentences. Acoustic differences were controlled by creating 
a single "neutral stress" version of the words (PERMIT). The authors expected RTs to be shorter when the target phoneme occurred on a syllable that had been predicted to be stressed based on the preceding rhythmic context. However, this was not the case, suggesting that the difference in RTs found by Shields et al. (1974) might in fact have been due to the stressed syllables' acoustic saliency. Nonetheless, while both Shields et al. (1974) and Pitt and Samuel (1990) based their hypotheses on theories of metrical grids, the meter of the sentences was not itself controlled for; additionally, factors other than stress rhythm may have confounded the effects of timing expectancies (e.g., semantic and syntactic prediction for whether a verb or a noun would occur in Pitt and Samuel, 1990). Thus it is hard to tell from these early studies whether rhythmic predictions are indeed at play in speech.

Subsequent studies induced temporal expectations for stress through the periodic or semi-periodic alternation of stressed and unstressed syllables (e.g., Pitt and Samuel, 1990, Exp. 2; Quené and Port, 2005; Schmidt-Kassow and Kotz, 2009a,b; Rothermich et al., 2012; Rothermich and Kotz, 2013). In their second experiment, Pitt and Samuel (1990) embedded neutral stress targets in strings of bisyllabic words presenting the same or opposite stress pattern as the target - either trochaic $(\mathrm{S}-\mathrm{w})$ or iambic $(\mathrm{w}-\mathrm{S})$. While in this case RTs were shorter for syllables predicted to be stressed based on the preceding rhythm, in their discussion Pitt and Samuel (1990) cast doubt on whether this result would generalize to natural sentences. One study using a similar methodology found that precise timing regularity (isochrony of stresses), rather than consistency in the metric pattern of the target and the preceding words, best explains differences in RTs (Quené and Port, 2005). This speaks against the claim that stress periodicity is primarily a perceptual phenomenon, suggesting that rhythmic predictions for stress are most reliably induced by physical periodicity of the stimulus which is seldom found in natural speech (though see Otterbein et al., 2012).

Nonetheless, recent studies provide evidence for rhythmic expectancies induced by sentences comprised of bisyllabic words with consistent trochaic or iambic patterns, but lacking exact isochrony. Schmidt-Kassow and Kotz (2009b) observed eventrelated brain potentials to "metric violations" induced by having a target word be pronounced with incorrect lexical stress (e.g., with a trochaic pattern rather than the correct iambic pattern) within such sentences. A biphasic pattern was observed, consisting of an early anterior negativity and a P600 effect. Schmidt-Kassow and Kotz (2009a) found similar results when using correctly pronounced target words with a stress pattern opposite to the preceding context (although this effect was only present when listeners were instructed to actively pay attention to the meter of the sentences). Additionally, Rothermich et al. (2012) observed a reduced N400 to semantically unpredictable target words when the words were embedded in sentences with regular (iambic or trochaic) rather than irregular stress patterns. This suggests that temporal regularity of stress in speech leads to expectations which in turn may facilitate semantic integration of unpredicted words. Lastly, eye-tracking evidence for the prediction of lexical stress was found for stimuli with a highly constraining metrical structure (limericks; Breen and Clifton, 2011). These experiments corroborate evidence from phoneme monitoring, suggesting that the alternation of stressed and unstressed syllables may contribute to timing expectancies for stress, though they do not speak to whether such predictions may be at play in naturally occurring speech.

\section{ENTRAINMENT}

The ABH (Shields et al., 1974; Pitt and Samuel, 1990) suggests that attention is directed to the predicted location of a stress regardless of how this location is predicted. Later studies, inducing rhythmic expectancies through periodicity, shifted their theoretical approach to the more specific notion of neural entrainment, as assumed in Dynamic Attending Theory (DAT; Large and Kolen, 1994; Large and Jones, 1999). This theory, which was primarily developed to understand the perception of musical rhythm, posits that listeners form expectations for when a beat will occur thanks to the entrainment, or synchronization, of their own neural oscillations with an external periodic stimulus. This leads to the dynamic allocation of attention to specific points in time. Neural oscillations have been shown to entrain to rhythmically organized stimuli (Lakatos et al., 2008), and to the strong beats of an imagined meter imposed over a periodic series of acoustically equal beats (Nozaradan et al., 2011).

While many have posited that this mechanism may also be involved in the perception of stress patterns in speech (e.g., Large and Jones, 1999; Port, 2003; Ghitza and Greenberg, 2009; Kotz and Schwartze, 2010; Goswami, 2012; Peelle and Davis, 2012), whether this is the case has not been unequivocally established. Cummins and Port (1998) found that in a speech cycling task (where a phrase was repeated multiple times in synchrony with a metronome), stresses tended to align with particular metrical positions in accordance with the principles of DAT (Port, 2003). However, it is not clear whether this work applies to regular speech and whether it translates to neural activity.

A promising lead comes from work positing a role for entrainment in the segmentation and temporal prediction of speech units at different timescales (for a review, see: Kösem and van Wassenhove, 2017; Meyer, 2017). Neural oscillations in the theta range $(4-8 \mathrm{~Hz})$ have been shown to synchronize with fluctuations in the temporal envelope of speech corresponding to the syllabic rate (Giraud and Poeppel, 2012; Peelle and Davis, 2012). Entrainment to the syllabic rate may be a fundamental mechanism for prediction, segmentation, and speech processing in general (Ghitza and Greenberg, 2009; Peelle and Davis, 2012). Oscillations in the delta range $(0.5-4 \mathrm{~Hz})$ have also been found to track the pitch contour of speech, possibly reflecting entrainment to intonational boundaries (Bourguignon et al., 2013). Entrainment is posited to be a fundamental element of neural mechanisms supporting the representation of temporal structure and temporal predictions in speech (Kotz and Schwartze, 2010; Schwartze and Kotz, 2013). To the best of our knowledge, however, neural entrainment to hierarchically organized stress patterns in speech remains to be empirically established. 


\section{PREDICTION IN EVERYDAY SPEECH}

The evidence for temporal prediction of stress reviewed thus far has relied on stimuli that contain a certain degree of periodicity, induced either through perfect isochrony (Quené and Port, 2005) or through the regular alternation of stressed and unstressed syllables (Pitt and Samuel, 1990; Schmidt-Kassow and Kotz, 2009a,b; Rothermich et al., 2012). The neural mechanism most commonly associated with these findings is the entrainment of neural oscillations to an external stimulus, in this case the pattern of stresses in speech. While entrainment may not require exact isochrony and can adjust to various types of rhythmic irregularity (Large and Jones, 1999; Large et al., 2002), it is not clear whether the stress patterns of natural speech present the coherence required by neural oscillations to entrain (Schwartze and Kotz, 2013). Schwartze and Kotz (2013) note that different types of speech may present more or less rhythmic regularity, and may therefore engage entrainment to various degrees.

Whether everyday speech presents enough rhythmic regularity to induce temporal expectations for stress through neural entrainment remains an open question. It is likely that neural entrainment is at play in the presently reviewed literature, as these studies induced expectancies through the periodic (or semi-periodic) presentation of stress. However, their generalization to the perception of natural speech remains to be tested. Given that natural speech presents far less periodicity than the stimuli utilized in these studies, two possibilities arise: (1) the prediction of stress in natural speech is reduced or non-existent, (2) mechanisms other than neural entrainment are involved in the prediction of stress. These two possibilities and their implications are explored in the following sections.

\section{No Prediction?}

It is possible that the prediction of stress observed in the studies here reviewed relies on general beat perception processes not typically utilized in speech perception. In other words, listeners may indeed entrain to the beat induced by the periodic presentation of stressed syllables, but the same phenomenon could have been induced by any other periodic stimulus (such as a simple sequence of beats, as in Cason and Schön, 2012). These predictions may therefore be tied to the specific manipulations of these experiments and not be present in everyday speech perception. This reflects the observation that the role of prediction mechanisms in language comprehension may have been overestimated through the use of overly predictable stimuli (Huettig and Mani, 2016).

However, it is unlikely that no prediction of stress is involved in natural speech. In the absence of prediction, any difference in RTs to stressed as opposed to unstressed syllables would be due to bottom-up, salient features of stressed syllables. This, however, is inconsistent with the finding that shorter RTs are shown even for syllables predicted to be stressed in absence of acoustic differences (Cutler, 1976; Cutler and Fodor, 1979). Additionally, it is not clear why phenomena such as stress shift would constrain the stress patterns of speech into hierarchically organized structures allowing prediction. Finally, the hierarchical nature of stress patterns suggests that prediction for the location of the strongest stress in a sentence (i.e., the nuclear stress) is required in order not to misclassify a pre-nuclear (relatively weaker) stress as nuclear (Calhoun, 2010). Thus, it is likely that mechanisms other than neural entrainment are involved in the prediction of stress for everyday speech.

\section{Other Forms of Prediction}

While the majority of studies have induced temporal predictions of stress by controlling the periodicity of the preceding speech, a few experiments have achieved similar results through different manipulations. Prosodically, the prediction of stress has been induced through a sentence's intonational contour (Cutler, 1976), as well as through the manipulation of the duration and the pitch quality of vowels preceding a target word (Brown et al., 2016).

This points to the idea that stress patterns may be better conceptualized in conjunction with other prosodic features such as intonation, rather than solely through fluctuations in amplitude envelope (e.g., as in Peelle and Davis, 2012). Special qualities in amplitude, pitch, duration, and spectral tilt are all thought to contribute to the perception of stress (Fry, 1955; Shattuck-Hufnagel and Turk, 1996; Sluijter and van Heuven, 1996; Breen et al., 2010). As context-dependent predictions have been observed for prosodic elements such as pitch accents (Weber et al., 2006; Dimitrova et al., 2012), prosodic boundaries (Clifton et al., 2002), and prominence shifts (Klassen and Wagner, 2017), these elements may need to be modulated as well, or at least controlled for, in order to fully understand the prediction of stress.

Moreover, the acoustic realization of a sentence's stress pattern may result from the interplay of several linguistic constraints that potentially influence language production (Calhoun, 2010). These include a tendency for rhythm, but also lexical and syntactic constraints, sentence focus, and information structure, as well as unplanned disfluencies and pauses (Ferreira, 2007). This is supported by studies that induced stress expectations through syntactic (Breen and Clifton, 2011) and information structural predictions (Cutler and Fodor, 1979). In this framework, the position of each stress results from the probabilistic alignment of these constraints with an overall metrical structure (Calhoun, 2010). Prediction at these different levels (e.g., semantic predictions) may therefore contribute to the prediction of stress. And, relatedly, predicting the timing of stressed syllables may be just one of many tools that listeners have for segmentation (Sanders and Neville, 2000) and for allocating attentional resources to new information (Cutler and Fodor, 1979), and may itself be secondary to other mechanisms in everyday speech.

\section{CONCLUSION}

In this review, we have focused on studies that induced temporal expectations for specific stress patterns based on the idea that stress in speech is rhythmically organized. However, while these studies have often appealed to theories of hierarchical metrical grids, they have typically induced prediction of stress by artificially increasing the amount of periodicity found in speech. 
While their results are consistent with the notion of neural entrainment involved in musical beat perception, whether this mechanism could account for the prediction of stress in natural speech - presenting far less periodicity - has not been established. We propose that other mechanisms may be at play, such as the prediction of other linguistic features that often coincide with perceived stress.

Nonetheless, the current literature offers insights into the way the prediction of stress through neural entrainment may be utilized for types of speech that naturally present higher degree of rhythmic regularity. For example, infant-directed speech and song present enhanced hierarchical temporal structure than the same materials directed at adults (Falk and Kello, 2017), and child-directed nursery rhymes display hierarchical amplitude modulations that may facilitate the development of phonological awareness through entrainment (Leong and Goswami, 2015). Thus, it is possible that speakers increase the regularity of their own speech to make it easier for listeners to entrain to and predict their stress patterns, consequently rendering speech more intelligible and facilitating language acquisition. Moreover, failure to recruit entrainment mechanisms during development may be fundamentally tied to language deficits such as developmental language disorder and developmental dyslexia (Goswami,

\section{REFERENCES}

Abercrombie, D. (1967). Elements of General Phonetics. Chicago, IL: Aldine Publishing Company.

Altman, G., and Carter, D. (1989). Lexical stress and lexical discriminability: stressed syllables are more informative, but why? Comput. Speech Lang. 3, 265-275. doi: 10.1016/0885-2308(89)90022-3

Aylett, M., and Turk, A. (2004). The smooth signal redundancy hypothesis: a functional explanation for relationships between redundancy, prosodic prominence, and duration in spontaneous speech. Lang. Speech 47, 31-56. doi: 10.1177/00238309040470010201

Bourguignon, M., De Tiège, X., De Beeck, M. O., Ligot, N., Paquier, P., Van Bogaert, P., et al. (2013). The pace of prosodic phrasing couples the listener's cortex to the reader's voice. Hum. Brain Mapp. 34, 314-326. doi: 10.1002/hbm. 21442

Breen, M., and Clifton, C. Jr. (2011). Stress matters: effects of anticipated lexical stress on silent reading. J. Mem. Lang. 64, 153-170. doi: 10.1016/j.jml.2010.11. 001.Stress

Breen, M., Fedorenko, E., Wagner, M., and Gibson, E. (2010). Acoustic correlates of information structure. Lang. Cogn. Process. 25, 1044-1098. doi: 10.1080/ 01690965.2010.504378

Brown, M., Salverda, A., Dilley, L., and Tanenhaus, M. K. (2016). Metrical expectations from preceding prosody influence perception of lexical stress. J. Exp. Psychol. 41, 306-323. doi: 10.1037/a0038689.Metrical

Calhoun, S. (2010). How does informativeness affect prosodic prominence? Lang. Cogn. Process. 25, 1099-1140. doi: 10.1080/01690965.2010.491682

Cason, N., and Schön, D. (2012). Rhythmic priming enhances the phonological processing of speech. Neuropsychologia 50, 2652-2658. doi: 10.1016/j. neuropsychologia.2012.07.018

Clifton, C. Jr., Carlson, K., and Frazier, L. (2002). Informative prosodic boundaries. Lang. Speech 45, 87-114. doi: 10.1177/00238309020450020101

Cummins, F., and Port, R. F. (1998). Rhythmic constraints on stress timing in English. J. Phon. 26, 145-171. doi: 10.1006/jpho.1998.0070

Cutler, A. (1976). Phoneme-monitoring reaction time as a function of intonation contour. Percept. Psychophys. 20, 55-60. doi: 10.3758/BF03198706

Cutler, A., and Fodor, J. A. (1979). Semantic focus and sentence comprehension. Cognition 7, 49-59. doi: 10.1016/0010-0277(79)90010-6
2011). Future studies should aim to establish the degree to which the mechanisms utilized for beat perception in music are applied to different types of speech and in different populations.

\section{AUTHOR CONTRIBUTIONS}

EB and FF contributed equally to the conceptualization of the arguments and preparation of this manuscript. The initial draft was prepared by EB.

\section{FUNDING}

The authors acknowledge support from the National Science Foundation GRFP number 1650042 awarded to EB and National Science Foundation research grant BCS-1650888 awarded to FF.

\section{ACKNOWLEDGMENTS}

The authors would like to thank the two reviewers for their helpful comments.

Cutler, A., and Foss, D. J. (1977). On the role of sentence stress in sentence processing. Lang. Speech 20, 1-10. doi: 10.1177/002383097702000101

Dauer, R. M. (1983). Stress-timing and syllable-timing reanalyzed. J. Phon. 11, 51-62.

Dimitrova, D. V., Stowe, L. A., Redeker, G., and Hoeks, J. C. J. (2012). Less is not more: neural responses to missing and superfluous accents in context. J. Cogn. Neurosci. 24, 2400-2418. doi: 10.1162/jocn_a_00302

Falk, S., and Kello, C. T. (2017). Hierarchical organization in the temporal structure of infant-direct speech and song. Cognition 163, 80-86. doi: 10.1016/j.cognition. 2017.02.017

Federmeier, K. D., and Kutas, M. (1999). A rose by any other name: longterm memory structure and sentence processing. J. Mem. Lang. 41, 469-495. doi: 10.1006/jmla.1999.2660

Ferreira, F. (1993). The creation of prosody during sentence production. Psychol. Rev. 100, 233-253. doi: 10.1037/0033-295X.100.2.233

Ferreira, F. (2007). Prosody and performance in language production. Lang. Cogn. Process. 22, 1151-1177. doi: 10.1080/01690960701461293

Fry, D. B. (1955). Duration and intensity as physical correlates of linguistic stress. J. Acoust. Soc. Am. 27, 765-768. doi: 10.1121/1.1908022

Ghitza, O., and Greenberg, S. (2009). On the possible role of brain rhythms in speech perception: intelligibility of time-compressed speech with periodic and aperiodic insertions of silence. Phonetica 66, 113-126. doi: 10.1159/00020 8934

Giraud, A., and Poeppel, D. (2012). Cortical oscillations and speech processing: emerging computational principles and operations. Nat. Neurosci. 15, 511-517. doi: $10.1038 / \mathrm{nn} .3063$

Goswami, U. (2011). A temporal sampling framework for developmental dyslexia. Trends Cogn. Sci. 15, 3-10. doi: 10.1016/j.tics.2010.10.001

Goswami, U. (2012). Entraining the brain: applications to language research and links to musical entrainment. Empir. Musicol. Rev. 7, 57-63. doi: 10.18061/ $1811 / 52980$

Gow, D., and Gordon, P. (1993). Coming to terms with stress: effects of stress location in sentence processing. J. Psycholinguist. Res. 22, 545-578.

Hayes, B. (1983). A grid-based theory of English meter. Linguist. Inq. 14, 357-393. Huettig, F., and Mani, N. (2016). Is prediction necessary to understand language? Probably not. Lang. Cogn. Neurosci. 31, 19-31. doi: 10.1080/23273798.2015. 1072223 
Klassen, J., and Wagner, M. (2017). Prosodic prominence shifts are anaphoric. J. Mem. Lang. 92, 305-326. doi: 10.1016/j.jml.2016.06.012

Kösem, A., and van Wassenhove, V. (2017). Distinct contributions of lowand high-frequency neural oscillations to speech comprehension. Lang. Cogn. Neurosci. 32, 536-544. doi: 10.1080/23273798.2016.1238495

Kotz, S. A., and Schwartze, M. (2010). Cortical speech processing unplugged: a timely subcortico-cortical framework. Trends Cogn. Sci. 14, 392-399. doi: 10.1016/j.tics.2010.06.005

Kuperberg, G. R., and Jaeger, F. (2016). What do we mean by prediction in language comprehension? Lang. Cogn. Neurosci. 31, 32-59. doi: 10.1080/23273798.2015. 1102299. What

Lakatos, P., Karmos, G., Mehta, A. D., Ulbert, I., and Schroeder, C. E. (2008). Entrainment of neuronal oscillations as a mechanism of attentional selection. Science 320, 110-113. doi: 10.1126/science.1154735

Large, E. W., Fink, P., and Kelso, J. A. S. (2002). Tracking simple and complex sequences. Psychol. Res. 66, 3-17. doi: 10.1007/s004260100069

Large, E. W., and Jones, M. R. (1999). The dynamics of attending: how people track time-varying events. Psychol. Rev. 106, 119-159. doi: 10.1037/0033295X.106.1.119

Large, E. W., and Kolen, J. F. (1994). Resonance and the perception of musical meter. Conn. Sci. 6, 177-208. doi: 10.1080/09540099408915723

Lehiste, I. (1977). Isochrony reconsidered. J. Phon. 5, 253-263.

Leong, V., and Goswami, U. (2015). Acoustic-emergent phonology in the amplitude envelope of child-directed speech. PLoS One 10:e014441. doi: 10.1371/journal.pone.0144411

Liberman, M., and Prince, A. (1977). On stress and linguistic rhythm. Linguist. Inq. 8, 249-336.

London, J. (2012). Three things linguists need to know about rhythm and time in music. Empir. Musicol. Rev. 7, 5-11. doi: 10.18061/1811/52973

Martin, J. G. (1972). Rhythmic (hierarchical) versus serial structure in speech and other behavior. Psychol. Rev. 79, 487-509. doi: 10.1037/h0033467

Meyer, L. (2017). The neural oscillations of speech processing and language comprehension: state of the art and emerging mechanisms. Eur. J. Neurosci. doi: 10.1111/ijlh.12426 [Epub ahead of print].

Morton, J., Marcus, S., and Frankish, C. (1976). Perceptual centers (P-centers). Psychol. Rev. 83, 405-408. doi: 10.1037/0033-295X.83.5.405

Nazzi, T., and Ramus, F. (2003). Perception and acquisition of linguistic rhythm by infants. Speech Commun. 41, 233-243. doi: 10.1016/S0167-6393(02)00106-1

Norris, D., McQueen, J. M., and Cutler, A. (1995). Competition and segmentation in spoken-word recognition. J. Exp. Psychol. 21, 1209-1228. doi: 10.1037/02787393.21.5.1209

Nozaradan, S., Peretz, I., and Missal, M. (2011). Tagging the neuronal entrainment to beat and meter. J. Neurosci. 31, 10234-10240. doi: 10.1523/JNEUROSCI. 0411-11.2011

Otterbein, S., Abel, C., Heinemann, L. V., Kaiser, J., and Schmidt-Kassow, M. (2012). P3b reflects periodicity in linguistic sequences. PLoS One 7:e51419. doi: 10.1371/journal.pone.0051419

Patel, A. (2008). Music, Language and the Brain. Oxford: Oxford UP.

Patel, A. (2011). Musical rhythm, linguistic rhythm, and human evolution. Music Percept. 24, 99-104. doi: 10.1525/rep.2008.104.1.92.This

Peelle, J. E., and Davis, M. H. (2012). Neural oscillations carry speech rhythm through to comprehension. Front. Psychol. 3:320. doi: 10.3389/fpsyg.2012. 00320
Pickering, M. J., and Garrod, S. (2007). Do people use language production to make predictions during comprehension? Trends Cogn. Sci. 11, 105-110. doi: 10.1016/j.tics.2006.12.002

Pitt, M. A., and Samuel, A. G. (1990). The use of rhythm in attending to speech. J. Exp. Psychol. 16, 564-573. doi: 10.1037/0096-1523.16.3.564

Port, R. F. (2003). Meter and speech. J. Phon. 31, 599-611. doi: 10.1016/j.wocn. 2003.08.001

Quené, H., and Port, R. F. (2005). Effects of timing regularity and metrical expectancy on spoken-word perception. Phonetica 62, 55-67. doi: 10.1159/ 000087222

Rothermich, K., and Kotz, S. A. (2013). Predictions in speech comprehension: FMRI evidence on the meter-semantic interface. Neuroimage 70, 89-100. doi: 10.1016/j.neuroimage.2012.12.013

Rothermich, K., Schmidt-Kassow, M., and Kotz, S. A. (2012). Rhythm's gonna get you: regular meter facilitates semantic sentence processing. Neuropsychologia 50, 232-244. doi: 10.1016/j.neuropsychologia.2011.10.025

Sanders, L. D., and Neville, H. J. (2000). Lexical, syntactic, and stress-pattern cues for speech segmentation. J. Speech Lang. Hear. Res. 43, 1301-1321. doi: 10.1044/jslhr.4306.1301

Schmidt-Kassow, M., and Kotz, S. A. (2009a). Attention and perceptual regularity in speech. Neuroreport 20, 1643-1647. doi: 10.1097/WNR.0b013e3283 33b0c6

Schmidt-Kassow, M., and Kotz, S. A. (2009b). Event-related brain potentials suggest a late interaction of meter and syntax in the P600. J. Cogn. Neurosci. 21, 1693-1708. doi: 10.1162/jocn.2008.21153

Schwartze, M., and Kotz, S. A. (2013). A dual-pathway neural architecture for specific temporal prediction. Neurosci. Biobehav. Rev. 37, 2587-2596. doi: 10.1016/j.neubiorev.2013.08.005

Selkirk, E. O. (1984). Phonology and Syntax: The Relation between Sound and Structure. Cambridge, MA: MIT Press.

Shattuck-Hufnagel, S., and Turk, A. E. (1996). A prosody tutorial for investigators of auditory sentence processing. J. Psycholinguist. Res. 25, 193-247. doi: 10.1007/BF01708572

Shields, J. L., McHugh, A., and Martin, J. G. (1974). Reaction time to phoneme targets as a function of rhythmic cues in continuous speech. J. Exp. Psychol. 102, 250-255. doi: 10.1037/h0035855

Sluijter, A M., and van Heuven, V. J. (1996). Spectral balance as an acoustic correlate of linguistic stress. J. Acoust. Soc. Am. 100, 2471-2485. doi: 10.1121/1. 417955

Weber, A., Braun, B., and Crocker, M. W. (2006). Finding referents in time: eyetracking evidence for the role of contrastive accents. Lang. Speech 49, 367-392. doi: 10.1177/00238309060490030301

Conflict of Interest Statement: The authors declare that the research was conducted in the absence of any commercial or financial relationships that could be construed as a potential conflict of interest.

Copyright (C) 2018 Beier and Ferreira. This is an open-access article distributed under the terms of the Creative Commons Attribution License (CC BY). The use, distribution or reproduction in other forums is permitted, provided the original author(s) and the copyright owner are credited and that the original publication in this journal is cited, in accordance with accepted academic practice. No use, distribution or reproduction is permitted which does not comply with these terms. 